\title{
Diagnostik bullöser Autoimmundermatosen
}

\section{Diagnostic of Bullous Autoimmune Diseases}

M. Kasperkiewicz, C. Rose, D. Zillikens

Klinik für Dermatologie, Allergologie und Venerologie, Universität zu Lübeck

\section{Lernziel \\ $\nabla$}

Die Diagnose einer bullösen Autoimmundermatose ist oft nicht leicht zu stellen, sodass hierbei neben der Kenntnis verschiedener klinischer Differenzialdiagnosen diagnostische Methoden von Bedeutung sind, die für den Leser nachfolgend zusammenfassend dargestellt werden. Der Beitrag soll den Leser mit den verschiedenen immunologischen Verfahren bei der differenzialdiagnostischen Einordnung blasenbildender Autoimmundermatosen mit intraepidermaler, junktionaler bzw. dermolytischer Spaltbildung und dem Algorithmus des diagnostischen Vorgehens vertraut machen.

\section{Einleitung \\ $\nabla$}

Bullöse Autoimmundermatosen stellen eine Gruppe seltener und schwerer, zum Teil lebensbedrohlicher, organspezifischer Autoimmunerkrankungen dar, welche durch Autoantikörperbildung gegen desmosomale und hemidesmosomale Struktur- und Adhäsionsproteine gekennzeichnet sind ( $\bullet$ Abb. 1). Man unterscheidet 4 immunpathologisch unterschiedliche Hauptgruppen, welche sich aus der Lokalisation der Spaltbildung und den betroffenen Zielstrukturen ergeben. Hierzu zählen Pemphigus- und Pemphigoid-Erkrankungen, Epidermolysis bullosa acquisita und die Dermatitis herpetiformis Duhring ( $\bullet$ Tab. 1). Neben der klinischen Inspektion und der histopathologischen Untersuchung sind direkte und indirekte immunfluoreszenzoptische Untersuchungen sowie ELISA und Immunoblot wichtige diagnostische Methoden [1,2].

\section{Allgemeine Aspekte zur Diagnostik blasenbildender Autoimmun- dermatosen \\ $\nabla$ \\ Klinik}

Das klinische Bild bullöser Autoimmundermatosen kann sehr variieren und muss von anderen mit Blasenbildung einhergehenden Erkrankungen unterschieden werden. Bei den Differentialdiagnosen kommen ursächlich bakterielle und virale Infektionen, allgemein entzündliche Mechanismen sowie physikalisch-traumatische und genetische Faktoren in Betracht ( $\bullet$ Tab. 2).

Merke: Als wichtige Differenzialdiagnosen zu bullösen Autoimmundermatosen sind infektiöse, immunologische, physikalisch-traumatische und hereditäre Ursachen zu berücksichtigen.

Die klinischen Veränderungen blasenbildender Autoimmundermatosen sind in der Regel auf Haut und hautnahe Schleimhäute beschränkt und zeichnen sich durch Blasen und deren Folgen (Erosionen, Krusten, Superinfektion, Narben) aus. Oft besteht erheblicher Juckreiz. Insbesondere in der Prodromalphase der Erkrankung kann jedoch das klinische Bild unspezifisch sein, wobei Blasen fehlen und stattdessen für unterschiedlich lange Zeit juckende urtikarielle Plaques oder ekzematöse Hautveränderungen dominieren können. Da Blasen an der Mundschleimhaut rasch einreißen, sind dort meist nur Erosionen sichtbar. Somit sollte bei unklaren juckenden Dermatosen oder refraktären Mundschleimhauterosionen auch eine bullöse Autoimmundermatose in die Differentialdiagnostik eingeschlossen werden [3].

Merke: Bei unklaren juckenden Dermatosen oder refraktären Mundschleimhauterosionen sollte an eine bullöse Autoimmundermatose gedacht werden.
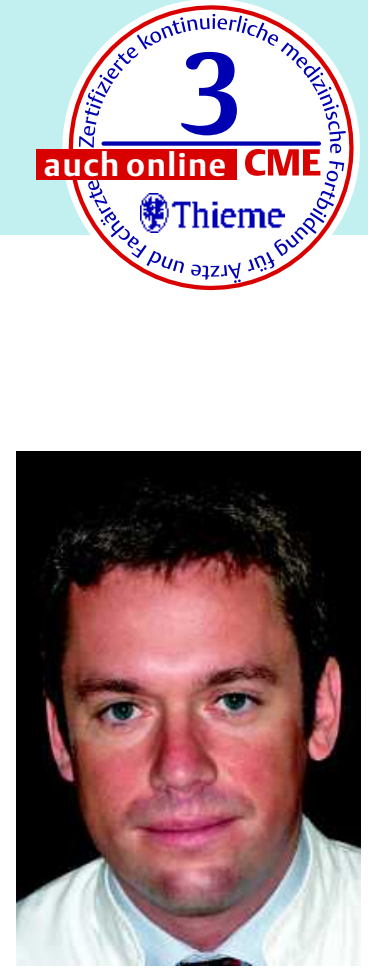

Dr. Michael Kasperkiewicz

Korrespondenzadresse Dr. Michael Kasperkiewicz Klinik für Dermatologie, Allergologie und Venerologie Universität zu Lübeck Ratzeburger Allee 160 23538 Lübeck Michael.Kasperkiewicz@ uk-sh.de

Bibliografie DOI $10.1055 / \mathrm{s}-2008-1077556$ Akt Dermatol 2008; 34: 301-312 c Georg Thieme Verlag KG Stuttgart · New York ISSN 0340-2541 


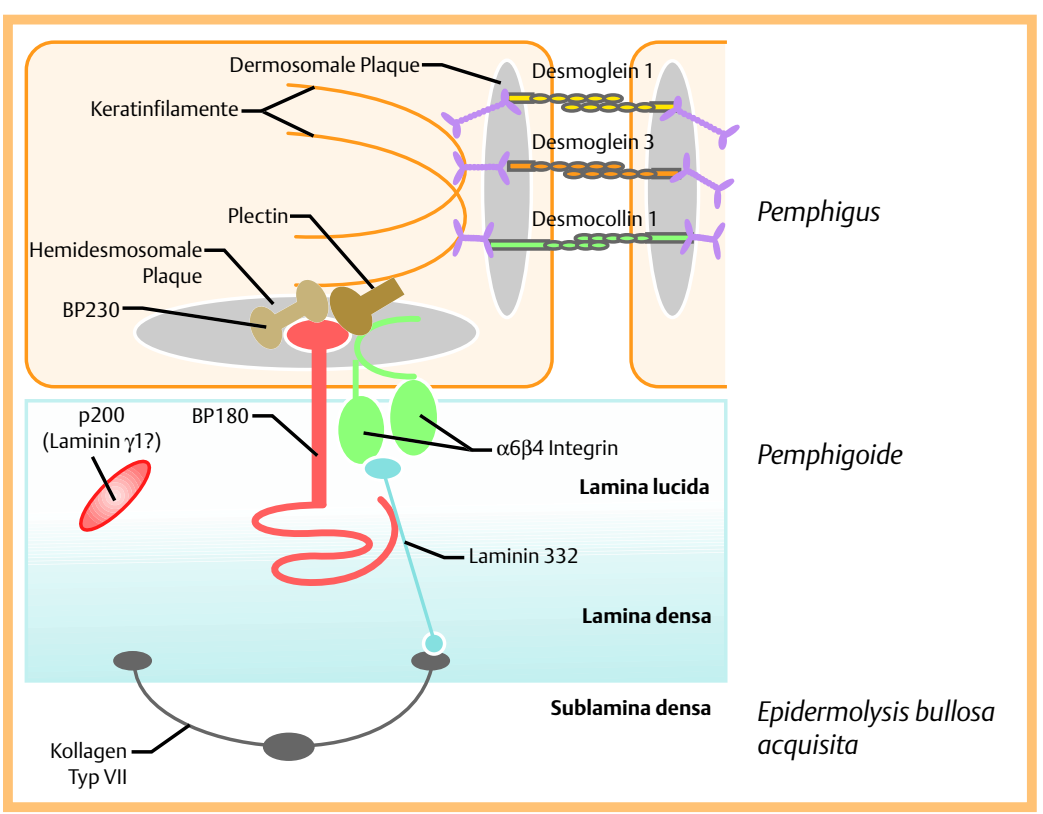

Abb. 1 Schematische Darstellung desmosomaler und hemidesmosomaler Strukturproteine. Desmosomen vermitteln den Zell-Zell-Kontakt innerhalb des Epithels. Über transmembranöse Proteine der Desmosomen, die Desmogleine und Desmocolline, wird die Interaktion zwischen zwei Keratinozyten hergestellt. Mit ihrem intrazellulären Anteil treten diese Proteine mit desmosomalen Plaqueproteinen in Kontakt, welche die Verbindung zu Keratinfilamenten mediieren. Hemidesmosomen dagegen verankern Epithelzellen auf der Basalmembran. Hierbei steht der intrazelluläre Anteil der transmembranösen Strukturproteine BP180 und $\alpha 6 \beta 4$-Integrin in Kontakt mit Proteinen der hemidesmosomalen Plaque (BP230 und Plectin), während sich der extrazelluläre Anteil der Ankerfilamente in die Lamina lucida der Basalmembran erstreckt. Über weitere Strukturproteine wie Laminin 332 wird ein Kontakt zu Verankerungsfibrillen (Kollagen Typ VII) hergestellt, die von der Lamina densa in die Sublamina densa der papillären Dermis reichen. p200 ist ein noch nicht vollständig charakterisiertes Autoantigen der unteren Anteile der Lamina lucida. Neure Arbeiten weisen darauf hin, dass p200 Laminin $\gamma 1$ entspricht. Auf der rechten Seite ist die Blasenbildungsebene bei den bullösen Autoimmunkrankheiten angedeutet. Pemphigus: intraepidermale Blasen; Pemphigoide: Blasen entlang der Lamina lucida; Epidermolysis bullosa acquisita: Blasen unterhalb der Lamina densa.

\section{Histologie}

Die histologische Untersuchung erkrankter Haut spielt bei der Diagnostik blasenbildender Autoimmundermatosen nur eine orientierende Rolle, kann jedoch dazu dienen, eine intraepidermale von einer subepidermalen Spaltbildung abzugrenzen. Bei fehlender Blasenbildung kann sie zudem durch Nachweis neutrophiler oder eosinophiler Granulozyten an der dermo-epidermalen Junktionszone (DEJ) oder in der Epidermis (eosinophile Spongiose) erste Hinweise auf eine bullöse Autoimmundermatose liefern. Nach immunfluoreszenzoptischem Ausschluss einer bullösen Autoimmundermatose sollte bei subepidermaler Blasenbildung differenzialdiagnostisch zum Beispiel an eine Arthropodenreaktion, eine Porphyrie oder eine bullöse Arzneimittelreaktion gedacht werden. Bei einer intraepithelialen Spaltbildung sind unter anderem eine bullöse Impetigo, ein M. Hailey-Hailey oder ein M. Darier in die Differentialdiagnose mit einzubeziehen. Für die histopathologische Untersuchung ist die komplette Entnahme eines möglichst frischen Bläschens oder die partielle Biopsie einer größeren Blase mit einem ausreichend großen anhängenden Hautareal zu empfehlen [4].

Hinweis: Für die Histologie sollte eine kleine Blase komplett oder eine größere Blase partiell entnommen werden.

Tab. 1 Immunpathologische Charakteristika bullöser Autoimmundermatosen.

\begin{tabular}{|c|c|c|c|c|}
\hline Erkrankung & Spaltbildung & Direkte Immunfluoreszenz & Indirekte Immunfluoreszenz & Zielantigene \\
\hline \multicolumn{5}{|l|}{ Pemphigus-Gruppe } \\
\hline Pemphigus vulgaris & $\begin{array}{l}\text { intraepidermal } \\
\text { suprabasal }\end{array}$ & interzellulär lgG, C3 & interzellulär lgG (Affenösophagus) & Dsg 3, Dsg 1 \\
\hline Pemphigus foliaceus & $\begin{array}{l}\text { intraepidermal } \\
\text { subkorneal }\end{array}$ & interzellulär lgG, C3 & interzellulär IgG (Affenösophagus) & Dsg 1 \\
\hline Paraneoplastischer Pemphigus & intraepidermal & interzellulär und linear IgG, C3 & interzellulär IgG (Rattenblase) & Plakine, Dsg 3, Dsg 1 \\
\hline IgA-Pemphigus & $\begin{array}{l}\text { intraepidermal } \\
\text { subkorneal }\end{array}$ & interzellulär IgA, C3 & interzellulär IgA (Affenösophagus) & Dsc 1, Dsg 3 \\
\hline \multicolumn{5}{|l|}{ Pemphigoid-Gruppe } \\
\hline Bullöses Pemphigoid & junktional & linear $C 3, \lg G$ an der DEJ & IgG epidermal (SSS) & BP180, BP230 \\
\hline Pemphigoid gestationis & junktional & linear C3, (IgG) an der DEJ & IgG epidermal (SSS) & BP180, BP230 \\
\hline Schleimhautpemphigoid & junktional & linear IgG, C3, IgA an der DEJ & IgG, IgA epidermal/dermal (SSS) & $\begin{array}{l}\text { BP180, } \\
\text { Laminin } 332 \text {, } \\
\alpha 6 \beta 4-\text { Integrin }\end{array}$ \\
\hline Lineare IgA-Dermatose & junktional & linear IgA, (C3) an der DEJ & IgA epidermal (SSS) & LAD-1, BP180 \\
\hline Anti-p200-Pemphigoid & junktional & linear $\lg G, C 3$ an der DEJ & IgG dermal (SSS) & p200 \\
\hline $\begin{array}{l}\text { Epidermolysis bullosa } \\
\text { acquisita }\end{array}$ & dermolytisch & linear $\lg G, C 3,(\operatorname{Ig} A)$ an der $D E J$ & $\lg G,(\operatorname{Ig} A)$ dermal (SSS) & Kollagen Typ VII \\
\hline $\begin{array}{l}\text { Dermatitis herpetiformis } \\
\text { Duhring }\end{array}$ & junktional & $\begin{array}{l}\text { granulär IgA in der papillären } \\
\text { Dermis }\end{array}$ & $\begin{array}{l}\text { IgA gegen Endomysium } \\
\text { (Affenösophagus) }\end{array}$ & Transglutaminase \\
\hline
\end{tabular}

DEJ: Dermo-epidermale Junktionszone, SSS: Sodium chloride-split skin (Kochsalzspalthaut), Dsg: Desmoglein, Dsc: Desmocollin. 


\section{Direkte Immunfluoreszenz (DIF)}

Mit dieser Methode können an Gefrierschnitten nach Inkubation mit fluoreszenzmarkierten Antikörpern in vivo gebundene Immunreaktantien (IgG, IgM, IgA, Komplement C3) nachgewiesen werden. Die DIF gilt als Goldstandard zur Diagnose einer bullösen Autoimmundermatose und sichert diese auch dann, wenn sich hierfür keine typischen histologischen Veränderungen zeigen. Eine negative DIF schließt in der Regel eine bullöse Autoimmundermatose aus, sollte jedoch wiederholt werden, falls klinisches Bild und Histologie eine bullöse Autoimmundermatose weiterhin vermuten lassen. Für die DIF sollte gesund erscheinende (Schleim-) Haut (periläsional) entnommen werden, da in der Blase selbst Immunglobuline und Komplement durch die Entzündung in der Regel bereits abgebaut und nicht mehr nachweisbar sind. Das Biopsat darf für diese Untersuchung nicht in Formalinlösung fixiert, sondern muss unmittelbar nach Entnahme eingefroren werden. Alternativ kann die Hautprobe in einem Transportmedium (Michel's Medium), welches vom untersuchenden Labor zur Verfügung gestellt wird, versandt werden $[1,2]$.

Hinweis: Für die direkte Immunfluoreszenz sollte gesund erscheinende Haut in der Umgebung einer Blase biopsiert werden.

\section{Indirekte Immunfluoreszenz (IIF)}

Die im Serum der Patienten zirkulierenden Autoantikörper können mittels IIF auf Organschnitten menschlicher (nach Vorinkubation mit $1 \mathrm{M} \mathrm{NaCl}-$ Lösung) oder tierischer Haut (Affenösophagus, Affen- oder Rattenharnblase) mit fluoreszenzmarkierten Zweitantikörpern nachgewiesen werden. Durch Verdünnungsreihen lässt sich der Antikörpertiter bestimmen. Für die IIF ist die Zusendung einer ungekühlten Serumprobe mit normaler Post ausreichend [1,2].

\section{Immunoblot und ELISA}

Nach Sicherung der Diagnose einer bullösen Autoimmundermatose durch DIF und IIF ist der Nachweis der molekularen Spezifität der Autoantikörper anzustreben. Extrakte von Epidermis, Dermis und kultivierten Keratinozyten sowie rekombinante Formen der Zielantigene stehen hierzu zur Verfügung. Die mittels ELISA bestimmten Autoantikörperkonzentrationen im Serum von Pemphigus- und Pemphigoid-Patienten (Antikörper gegen Desmoglein 1 und 3 sowie BP180 NC16A) korrelieren meist gut mit der Krankheitsaktivität und eignen sich daher als Verlaufsparameter und zur Einschätzung des weiteren Therapiebedarfs $[1,2]$.

\begin{tabular}{|c|c|}
\hline Infektionserkrankungen & $\begin{array}{l}\text { Impetigo contagiosa } \\
\text { Staphylococcal-Scalded-Skin-Syndrom } \\
\text { Bullöses Erysipel } \\
\text { Herpes simplex } \\
\text { Varizellen } \\
\text { Herpes zoster } \\
\text { Hand-Fuß-Mund-Krankheit } \\
\text { Bullöse Skabies }\end{array}$ \\
\hline Immunologische Erkrankungen & $\begin{array}{l}\text { Bullöser systemischer Lupus erythematodes } \\
\text { Dyshidrosiformes Hand-/Fußekzem } \\
\text { Bullöse Arzneiexantheme (SJS, TEN) } \\
\text { Bullöse Iktusreaktion } \\
\text { Bullöser/erosiver Lichen ruber planus } \\
\text { Eythema exsudativum multiforme (EEM) } \\
\text { Bullöse Vaskulitis allergica } \\
\text { Bullöse Mastozytose } \\
\text { Bullöse Amyloidose } \\
\text { Subkorneale Pustulose (Sneddon-Wilkinson) }\end{array}$ \\
\hline Bullöse Autoimmundermatosen & $\begin{array}{l}\text { Pemphigus-Erkrankungen } \\
\text { Pemphigoid-Erkrankungen } \\
\text { Epidermolysis bullosa acquisita } \\
\text { Dermatitis herpetiformis Duhring }\end{array}$ \\
\hline Metabolische Erkrankungen & $\begin{array}{l}\text { Porphyria cutanea tarda } \\
\text { Bullosis diabeticorum }\end{array}$ \\
\hline $\begin{array}{l}\text { Physikalische/traumatische } \\
\text { Erkrankungen }\end{array}$ & $\begin{array}{l}\text { Friktion } \\
\text { Verbrennung/Verbrühung, Erfrierung, Verätzung } \\
\text { Dermatitis solaris } \\
\text { Dermatitis artefacta }\end{array}$ \\
\hline Hereditäre Erkrankungen & $\begin{array}{l}\text { Epidermolysis-bullosa-simplex-Gruppe } \\
\text { Junktionale Epidermolysis-bullosa-Gruppe } \\
\text { Dystrophische Epidermolysis-bullosa-Gruppe } \\
\text { M. Hailey-Hailey } \\
\text { Incontinentia pigmenti (Stadium 1) } \\
\text { Kindler-Syndrom } \\
\text { Kongenitale bullöse ichthyosiforme Erythrodermie }\end{array}$ \\
\hline Sonstige Erkrankungen & $\begin{array}{l}\text { M. Grover } \\
\text { Miliaria }\end{array}$ \\
\hline
\end{tabular}

\section{Diagnostische Charalteristika blasen- bildender Autoimmundermatosen $\nabla$}

\section{Pemphigus-Erkrankungen}

Pemphigus-Erkrankungen sind durch das Auftreten von Autoantikörpern gegen desmosomale Strukturproteine gekennzeichnet, welche zur Schädigung der intraepidermalen Kohärenz führen (intraepidermale Spaltbildung).

Beim Pemphigus vulgaris (PV) treten Blasen und meist schmerzhafte Erosionen vorwiegend an den Schleimhäuten auf, initial meist an der Mundschleimhaut ( $\bigcirc$ Abb.2a). Histologisch zeigt sich eine Akantholyse mit suprabasaler Spaltbildung. In der DIF finden sich vor allem in der unteren Epidermis interzelluläre IgG- und C3-Ablagerungen. In der IIF mit Affenösophagus lassen sich bei ca. 95\% der PV-Patienten zirkulierende Autoantikörper nachweisen ( $\bullet$ Abb. 2b). Die Autoantikörper reagieren im ELISA mit Desmoglein 3 und gelegentlich zusätzlich mit Desmoglein 1.

Der Pemphigus foliaceus (PF) betrifft im Gegensatz zum PV ausschließlich die verhornende Haut ( $\bigcirc$ Abb.3). Die Blasenbildung liegt subkorneal, und die Autoantikörper binden in der

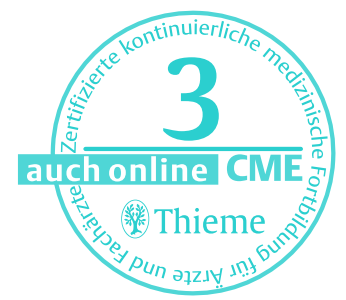


Abb. 2 a Pemphigus vulgaris mit Ulcera der Mundschleimhaut. b Indirekte Immunfluoreszenz auf Affenösophagus beim Pemphigus. IgG-Serumantikörper binden mit einem interzellulären Muster am Ösophagusepithel.

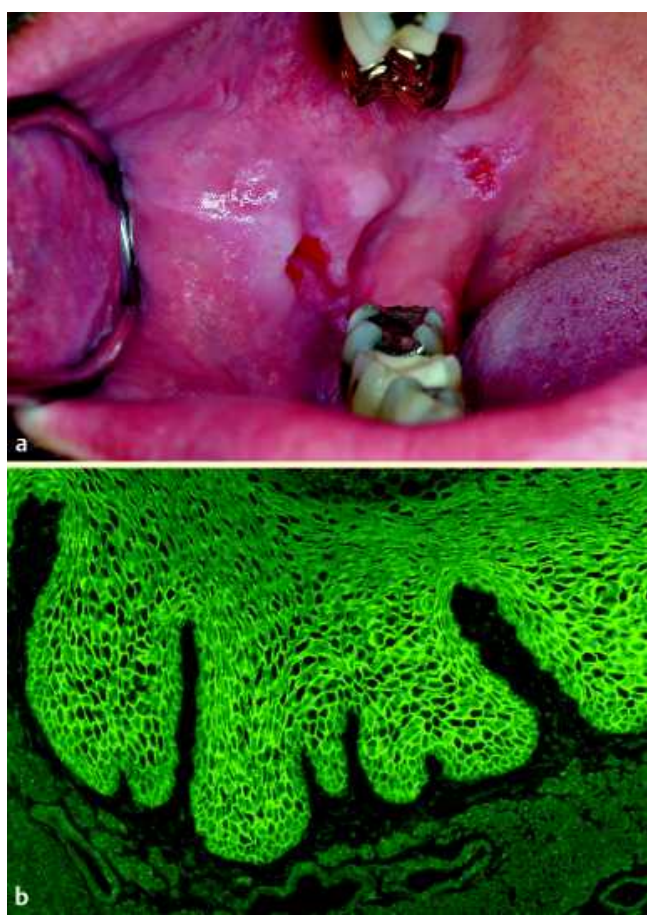

Abb. 3 Pemphius foliaceus mit konfluierenden Erythemen und Erosionen.

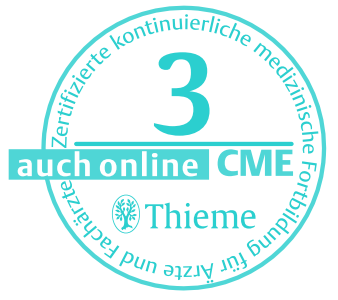

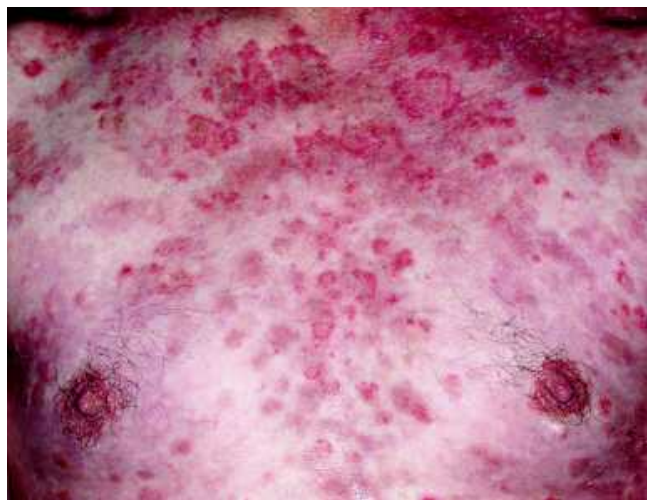

DIF vor allem an die oberen Epidermisschichten. Das Zielantigen dieser Autoantikörper ist Desmoglein 1. Die im ELISA ermittelten Serumspiegel der Autoantikörper gegen Desmoglein 1 und/ oder 3 korrelieren eng mit der Krankheitsaktivität des Pemphigus und sind als Verlaufsparameter geeignet.

Merke: Die im ELISA ermittelten Serumspiegel der Autoantikörper gegen Desmoglein 1 und/oder 3 korrelieren eng mit der Krankheitsaktivität des Pemphigus und sind als Verlaufsparameter geeignet.

Von PV und PF wird der paraneoplastische Pemphigus (PNP) abgegrenzt und stellt sowohl klinisch als auch immunpathologisch eine eigene Entität dar. Klinisch sieht man meist ausgedehnte Mundschleimhauterosionen und zusätzlich polymorphe Hautveränderungen mit oder ohne Blasen ähnlich einem Erythema exsudativum

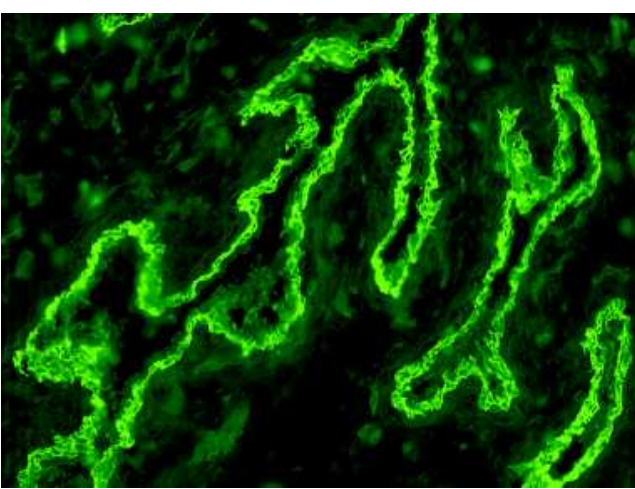

Abb. 4 Indirekte Immunfluoreszenz auf Rattenharnblase beim paraneoplastischen Pemphigus. Interzelluläre und zytoplasmatische Bindung von IgG-Serumantikörpern am Harnblasenepithel.

multiforme. Histologisch zeigt sich im typischen Fall eine Kombination aus einer vakuoligen Interface-Dermatitis und einer Akantholyse. In der DIF lassen sich Ablagerungen von IgG und C3 sowohl interzellulär als auch entlang der Basalmembran finden. Die Autoantikörper des PNP sind neben Desmoglein 1 und/oder 3 auch gegen verschiedene Plakine gerichtet. Im Gegensatz zu Desmoglein 1 und 3 werden Desmoplakine auch im Epithel der Harnblase exprimiert. Eine wichtige Untersuchung zur Diagnose eines PNP ist daher die IIF an Affen- oder Rattenharnblasenepithel als Substrat ( $\bullet$ Abb. 4). Der PNP ist obligat mit einer okkulten oder manifesten Neoplasie assoziiert, insbesondere häufig mit Non-Hodgkin-Lymphomen der B-Zell-Reihe.

Im Gegensatz zu den anderen Pemphigus-Erkrankungen finden sich bei dem seltenen IgAPemphigus Autoantikörper der IgA-Klasse, welche gegen Desmocollin 1 oder seltener Desmoglein 3 gerichtet sind [ $5-7]$.

\section{Pemphigoid-Erkrankungen}

Die Pemphigoid-Erkrankungen sind durch Autoantikörper gegen hemidesmosomale Strukturproteine gekennzeichnet. Dies führt zum Adhärenzverlust basaler Keratinozyten von der Basalmembran (junktionale Spaltbildung).

Das bullöse Pemphigoid (BP) stellt den häufigsten Vertreter der bullösen Autoimmundermatosen dar, betrifft vor allem ältere Menschen und ist klinisch durch juckende Hautveränderungen gekennzeichnet ( Abb.5a). Die Erkrankung kann über längere Zeit und auch gänzlich ohne Blasenbildung unter dem klinischen Bild eines Ekzems, einer Urtikaria oder einer Prurigo simplex subacuta verlaufen. Im Falle einer Blase zeigt sich histologisch eine subepidermale Spaltbildung, die in der Regel mit einer mehr oder weniger dichten Infiltration eosinophiler Granulozyten assoziiert ist. Die DIF zeigt lineare Ablagerungen von IgG und $\mathrm{C} 3$ an der $\mathrm{DEJ}(\odot \mathrm{Abb}$. $\mathbf{5}$ b). In der IIF auf $1 \mathrm{M} \mathrm{NaCl-gespaltener} \mathrm{humaner} \mathrm{Haut} \mathrm{las-}$ sen sich bei 80 bis $90 \%$ der Patienten zirkulieren- 

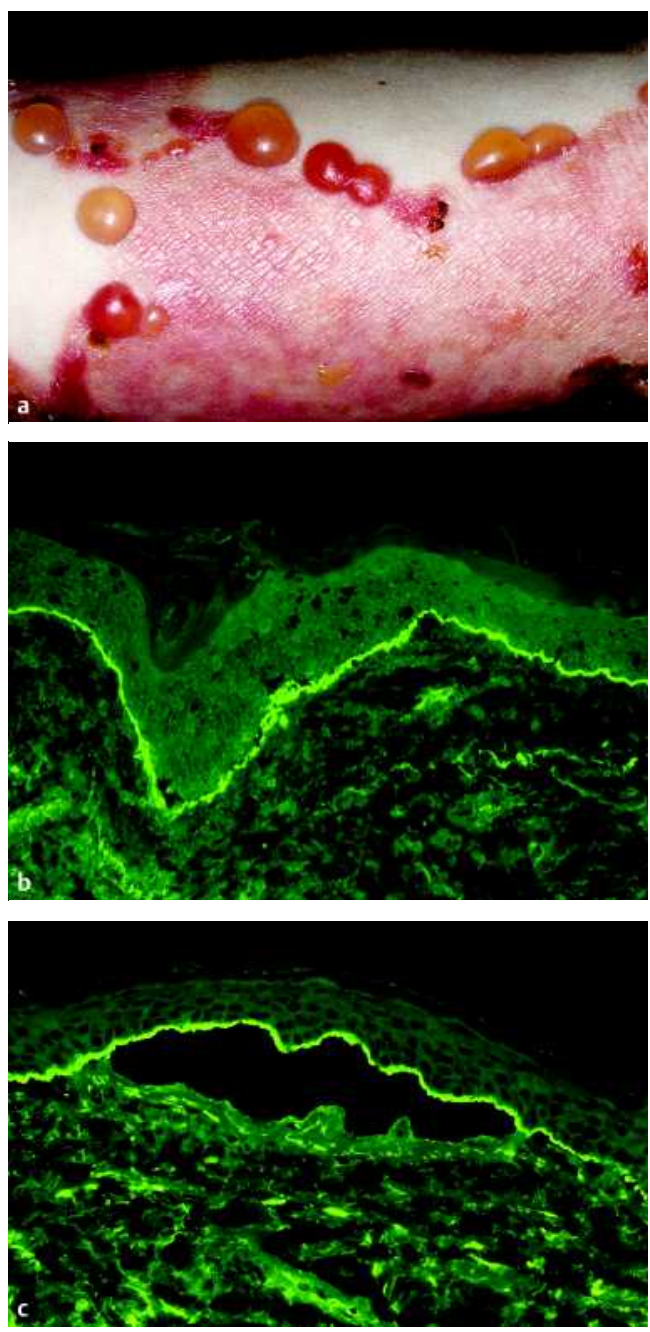

de Autoantikörper nachweisen, die im Dach der artifiziell erzeugten Blase binden ( $\bullet$ Abb.5c). Diese Autoantikörper sind gegen zwei Strukturproteine der Hemidesmosomen, BP180 und BP230, gerichtet und können mittels Immunoblot epidermaler Extrakte oder kultivierter Keratinozyten spezifiziert werden ( $\bullet$ Abb. 5d). Darüber hinaus stehen zwei kommerziell erhältliche sensitive ELISA-Systeme zur Verfügung (MBL, Nagoya, Japan; Euroimmun, Lübeck), mit denen die mit der Krankheitsaktivität korrelierenden Serumspiegel der Autoantikörper gegen die immundominante Domäne von BP180 (NC16A) nachgewiesen werden können.

Merke: Die Serumspiegel der Autoantikörper gegen die immundominante NC16A-Domäne von BP180 korrelieren im Gegensatz zu Autoantikörpern gegen BP230 mit der Krankheitsaktivität des BP.

Das Pemphigoid gestationis (PG) ist eine dem BP ähnliche, selbstlimitierte Schwangerschaftsdermatose, die sich meist in der zweiten Schwangerschaftshälfte oder auch unmittelbar postpartal

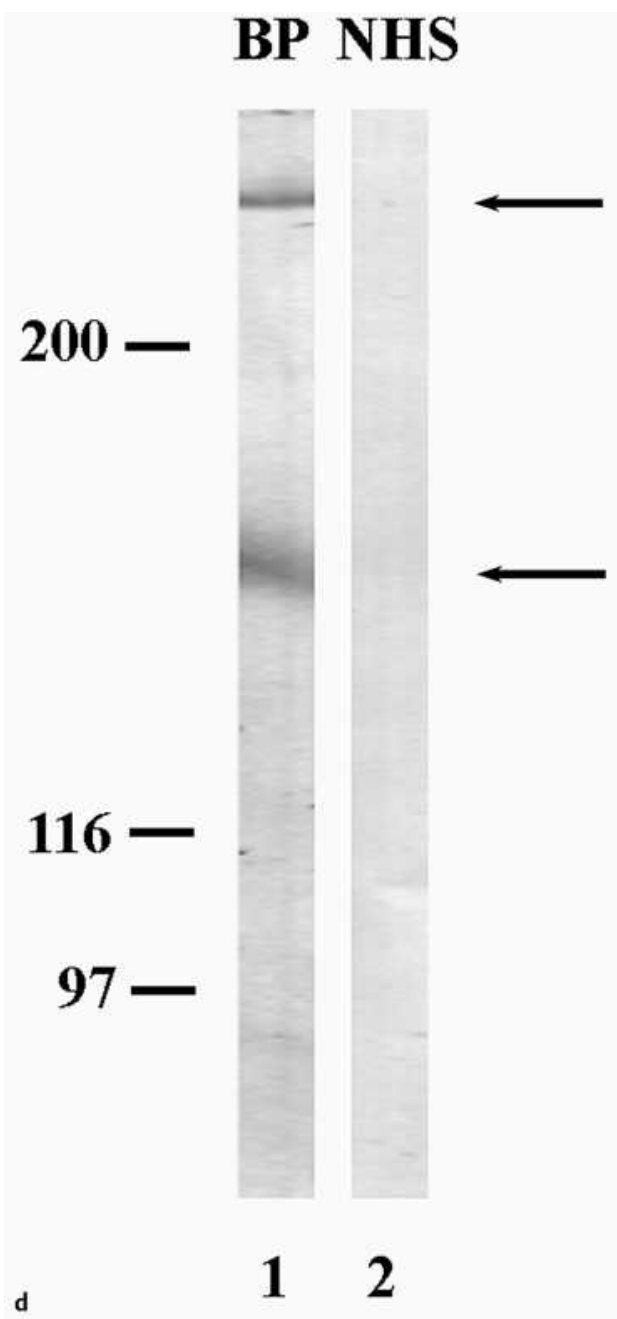

Abb. 5 a Bullöses Pemphigoid mit prallen, teils hämorrhagischen Blasen, Erosionen und Krusten. b Direkte Immunfluoreszenz periläsionaler Haut beim bullösen Pemphigoid. Lineare Ablagerung von C3 an der dermo-epidermalen Junktionszone. c Indirekte Immunfluoreszenz auf $1 \mathrm{M} \mathrm{NaCl}$-gespaltener humaner Haut beim bullösen Pemphigoid. IgGSerumantikörper binden an der epidermalen Seite des artifiziellen Spalts. d Immunoblot mit Keratinozytenextrakt beim bullösen Pemphigoid (BP). Keratinozytenextrakt wurde mittels 7,5\% SDS-PAGE aufgetrennt, auf Nitrozellulose übertragen und mit Patienten-Serum (Spur 1) und Serum eines gesunden Spenders (NHS; Spur 2) inkubiert. In Spur 1 reagiert das BP-Serum mit BP180 und BP230. Das Kontrollserum zeigt keine spezifische Reaktivität. Die Positionen der Molekulargewichtsmarker sind auf der linken Seite gezeigt, auf der rechten Seite (Pfeile) die von BP230 und BP180.

manifestiert. Das PG verläuft in der Regel ohne Blasen und präsentiert sich mit juckenden ekzematösen, urtikariellen oder papulösen Hautveränderungen ( $\bullet$ Abb. 6). In der DIF finden sich lineare C3- und geringer ausgeprägt IgG-Ablagerungen an der DEJ. Das PG kann in den meisten Fällen bereits durch eine serologische Untersuchung diagnostiziert werden. Gut geeignet ist hierfür der Komplementbindungstest, der sogenannte Herpes-gestationis-Test, bei dem in der IIF in einem weiteren Inkubationsschritt Komplement hinzugegeben wird. Während sich auf diese Weise in über $90 \%$ der Fälle Komplement-fixierende IgG1-Antikörper im Blasendach nachweisen lassen, werden bei der üblichen IIF mit Kochsalzspalthaut nur in 30\% zirkulierende Autoantikörper gefunden. Wie beim BP sind die Autoantikörper gegen BP180 NC16A gerichtet, und die Serumspiegel des ELISA korrelieren eng mit der Krankheitsaktivität.

Merke: Das Pemphigoid gestationis kann in den meisten Fällen bereits serologisch gesichert werden. 
Abb. 6 Pemphigoid gestationis bei einer Patientin im 3. Trimenon. Erythematöse Papeln und Plaques ohne Blasenbildung am Abdomen.

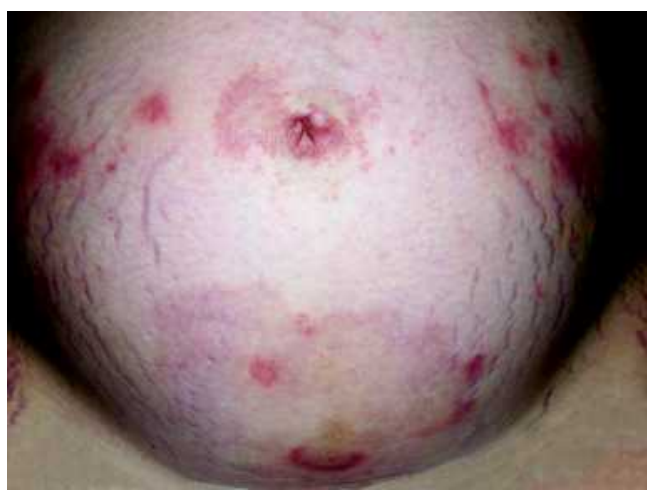

Das Schleimhautpemphigoid betrifft unterschiedliche Schleimhäute mit geschichtetem Plattenepithel und kann durch Vernarbung zu Blindheit sowie Atemwegs- und Ösophagusstrikturen führen. Die DIF zeigt lineare Ablagerungen von IgG, C3 oder IgA. Hierfür ist besonders die Wangenschleimhaut geeignet, auch wenn diese klinisch nicht betroffen ist. Mittels IIF lassen sich in ca. $50 \%$ der Fälle IgG- oder IgA-Autoantikörper nachweisen, die an die epidermale und/oder dermale Seite des artifiziellen Spalts binden. BP180, Laminin 332 (frühere Bezeichnung: Laminin 5) und

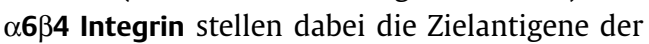
Autoantikörper dar. Antikörper gegen Laminin 332 und $\alpha 6 \beta 4$ Integrin werden mittels Immunoblot oder ELISA unter Verwendung extrazellulärer Matrixproteine kultivierter Keratinozyten nachgewiesen. Das Anti-Laminin 332-Pemphigoid ist in 30\% der Fälle mit Malignomen, insbesondere Adenokarzinomen assoziiert, sodass eine Tumorsuche durchgeführt werden sollte $[5,6]$.

Merke: Beim Schleimhautpemphigoid binden IgGund/oder IgA-Serumautoantikörper in der indirekten Immunfluoreszenz an die epidermale und/oder dermale Seite der $\mathrm{NaCl}$-separierten humanen Haut.

Eine klinisch dem BP verwandte, seltene, jedoch zunehmend häufiger diagnostizierte Pemphigoid-Erkrankung stellt das Anti-p200-Pemphigoid dar. Bei dieser Erkrankung zeigen sich in der DIF lineare IgG- und C3-Ablagerungen an der Basalmembran. In der IIF auf Spalthaut binden die $\mathrm{Au}-$ toantikörper am Blasenboden, und im Immunoblot mit Extrakten humaner Dermis findet sich eine Reaktivität mit einem 200-kDa-schweren Protein (p200) ( $\odot$ Abb. 7) [8]. Kürzlich vorgetragene Ergebnisse weisen darauf hin, dass die Antikörper dieser Erkrankung gegen die Laminin $\gamma 1$-Kette gerichtet sind [9].

Die lineare IgA-Dermatose ( $L A D)$ tritt sowohl im Erwachsenen- als auch im Kindesalter auf und stellt die häufigste bullöse Autoimmundermato-

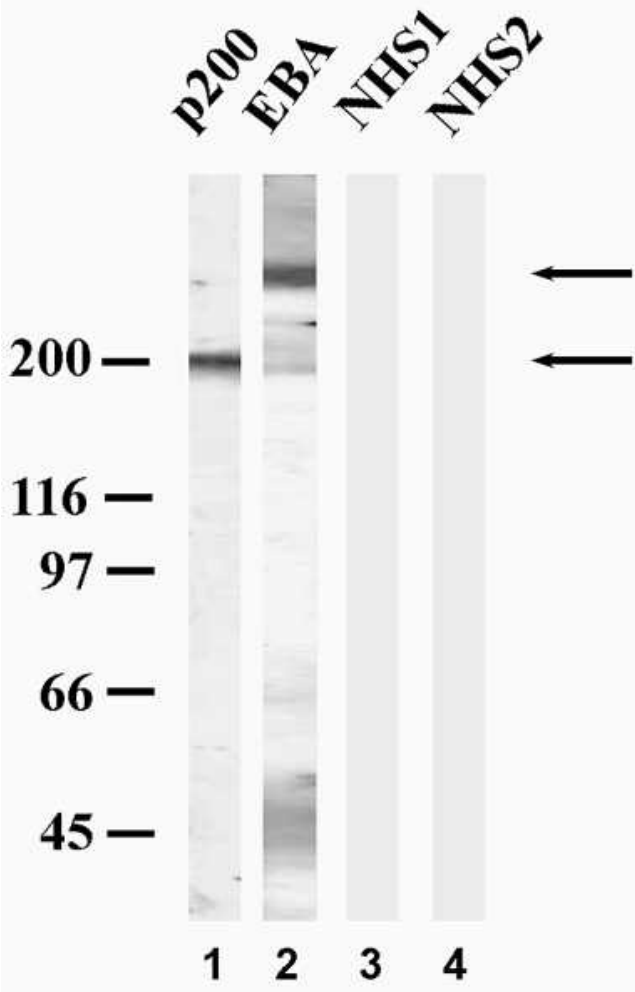

Abb. 7 Immunoblot mit dermalem Extrakt bei Anti-p200Pemphigoid und Epidermolysis bullosa acquisita (EBA). Dermales Extrakt wurde mittels 7,5\% SDS-PAGE separiert, auf Nitrozellulose übertragen und mit Serum eines Patienten mit Anti-p200-Pemphigoid (Spur 1), EBA (Spur 2) und zweier gesunder Spender (NHS1 und NHS2; Spur 3-4) inkubiert. Serumantikörper des Patienten mit Anti-p200Pemphigoid erkennen ein 200-kDa-schweres dermales Protein. Das Serum des Patienten mit EBA reagiert mit Kollagen Typ VII (290 kDa). Die Kontrollseren zeigen keine spezifische Bindung. Die Positionen der Molekulargewichtsmarker sind auf der linken Seite gezeigt, auf der rechten Seite (Pfeile) die von p200 und Kollagen Typ VII.

se des Kindesalters dar. Das klinische Bild ist vielfältig: Erytheme, urtikarielle Plaques, pralle Blasen, gruppiert stehende Bläschen wie bei der Dermatitis herpetiformis oder rosettenartig angeordnete Vesikel. Histologisch sieht man eine subepidermale Blase mit zahlreichen Neutrophilen und meist wenigen Eosinophilen. Die DIF zeigt lineare IgA-Ablagerungen an der DEJ. Auf humaner Spalthaut lassen sich bei $80 \%$ der Kinder und 30\% der Erwachsenen zirkulierende IgA-Autoantikörper nachweisen, die im Blasendach binden. Als Autoantigen wurde das extrazelluläre Spaltprodukt von BP180, ein 120-kDa-Protein (LAD-1), identifiziert. Für die Immunoblot-Untersuchung kann diese lösliche extrazelluläre Domäne von BP180 aus dem Zellkulturüberstand kultivierter Keratinozyten gewonnen werden $[10,11]$. 
Epidermolysis bullosa acquisita (EBA)

Bei der EBA unterscheidet man 3 klinische Varianten: mechanobullöse, inflammatorisch-generalisierte und Schleimhautpemphigoid-artige Form. Insbesondere bei der mechanobullösen Variante heilen die Hautveränderungen häufig unter Milienbildung ab. In der DIF lassen sich entweder lineare Ablagerungen von IgG und C3 oder seltener IgA (IgA-EBA) an der DEJ nachweisen, während in der IIF auf humaner Spalthaut bei ca. $50 \%$ der Patienten zirkulierende, gegen Kollagen Typ VII gerichtete IgG- oder IgA-Autoantikörper im Blasenboden binden ( $\boldsymbol{A}$ Abb. 8). Im Immunoblot reagieren Patientenautoantikörper mit einem 290-kD schweren Protein, das aus dermalem Extrakt gewonnen wird ( 0 Abb. 7). Außerdem steht der immundominante Abschnitt von Kollagen Typ VII, die NC1-Domäne, in rekombinanter Form zur Verfügung [10,11].

\section{Dermatitis herpetiformis (DH) Duhring}

Die DH ist eine überwiegend im mittleren $\mathrm{Er}$ wachsenenalter auftretende chronische, stark juckende Dermatose, welche typischerweise mit geröteten Papeln und Papulovesikeln an den Streckseiten der Extremitäten, sakral und am Kapillitium einhergeht. Die Erkrankung ist obligat mit einer glutensensitiven Enteropathie (Zöliakie) assoziiert und stellt die kutane Manifestation dieser intestinalen Erkrankung dar. Histologisch zeigt sich typischerweise eine subepidermale Blase mit neutrophilen Granulozyten, die Papillenabszesse ausbilden können. Voraussetzung zur Diagnose einer DH ist der Nachweis granulärer IgA-Ablagerungen in der papillären Dermis in der DIF, entweder diskontinuierlich in den Papillenspitzen oder kontinuierlich in der oberen Dermis entlang der DEJ ( $\bullet$ Abb. 9). In der IIF auf Affenösophagus finden sich Serumantikörper der IgA-Klasse gegen Endomysium. Diese Antikörper sind gegen das Enzym Gewebstransglutaminase gerichtet und lassen sich auch mittels ELISA detektieren. Der Nachweis dieser Antikörper sichert bei den Patienten die Diagnose einer Zöliakie. Zusätzlich lassen sich mittels ELISA Antikörper gegen die epidermale Transglutaminase nachweisen, deren Bedeutung für die Diagnostik der DH ist jedoch letztlich nicht geklärt [10,11].

Merke: Die direkte Immunfluoreszenz periläsionaler Haut zeigt bei der Dermatitis herpetiformis charakteristische granuläre IgA-Ablagerungen in der papillären Dermis.

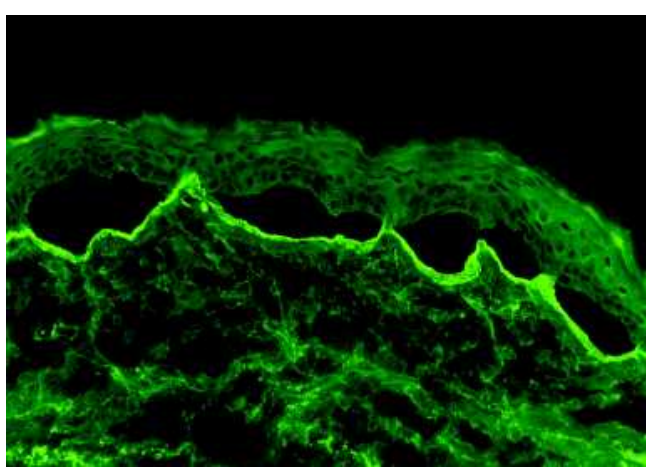

Abb. 8 Indirekte Immunfluoreszenz auf $1 \mathrm{M} \mathrm{NaCl}$-gespaltener humaner Haut bei der Epidermolysis bullosa acquisita. IgG-Serumantikörper binden an der dermalen Seite des artifiziellen Spalts.

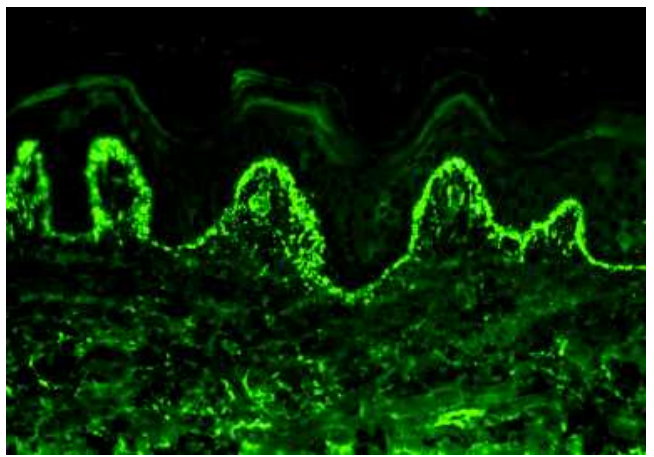

Abb. 9 Direkte Immunfluoreszenz periläsionaler Haut bei der Dermatitis herpetiformis Duhring. Kontinuierliche granuläre Ablagerungen von IgA in der papillären Dermis.

\section{Zusammenfassung}

$\nabla$

Entscheidend für die Diagnose einer bullösen Autoimmundermatose ist neben der Klinik und Histologie vor allem der Nachweis von Autoantikörpern in periläsionaler Haut und/oder im Serum der Patienten. In der Haut werden die Autoantikörper mittels direkter Immunfluoreszenz detektiert, für den Nachweis im Serum dient als Screening-Methode die indirekte Immunfluoreszenz auf Organschnitten. Der positive Nachweis in der direkten Immunfluoreszenz gilt als Goldstandard zur Diagnose einer bullösen Autoimmundermatose. Bei Patienten mit hohem klinischen und/oder histologischen Verdacht auf eine bullöse Autoimmundermatose kann versucht werden, die Diagnose ausschließlich serologisch zu sichern. Die genaue Charakterisierung der Spezifität der Serumantikörper gelingt durch sensitive Immunoblot- oder ELISA-Techniken unter Verwendung von Autoantigen-Extrakten oder rekombinanten Autoantigenen. Die Kenntnis der jeweiligen Autoantiköperspezifität ist von Bedeutung für die differenzialtherapeutischen Überlegungen. Die mittels ELISA oder Immunoblot bestimmte Autoantikörperkonzentration im Serum von Pemphigus- und Pemphigoid-Patienten korreliert meist gut mit der Krankheitsaktivität und eignet sich dadurch als Verlaufsparameter und zur Einschätzung des weiteren Therapiebedarfs. 


\section{Literatur}

1 Sitaru C, Goebeler M, Zillikens D. Bullöse Autoimmundermatosen (I): Pathogenese und Diagnostik. J Dtsch Dermatol Ges 2004; 2: 123 - 138

2 Kasperkiewicz M, Zillikens D, Rose C. Bullöse Autoimmundermatosen: Praktisches Vorgehen bei der Diagnostik. Derm 2007; 13: 352-361

3 Zillikens D. Bullöse Autoimmundermatosen. In: BraunFalco O, Plewig G, Wolff HH, Burgdorf W, Landthaler M, Hrsg: Dermatologie, 5. Auflage. Heidelberg Berlin: Springer, 2005: 607-638

4 Rose C, Bröcker EB, Zillikens D. Stellenwert der histologischen Untersuchung in der Diagnostik bullöser Autoimmundermatosen. J Dtsch Dermatol Ges 2004; 2: 96-104

5 Jainta S, Schmidt E, Bröcker EB, Zillikens D. Diagnostik und Therapie bullöser Autoimmunerkrankungen der Haut. Dt Ärztbl 2001; 98A : 1320-1325
6 Hertl M, Schuler G. Bullöse Autoimmundermatosen, Teil 3: Diagnostik und Therapie. Hautarzt 2002; 53: 352 366

7 Schmidt E, Bröcker EB, Zillikens D. Pemphigus: Verlust des desmosomalen Zell-Zell-Kontaktes. Hautarzt 2000; 51: 309-318

8 Shimanovich I, Rose C, Hirako Y, Butt-Dörje E, Zillikens D. Anti-p200-Pemphigoid - Eine neue blasenbildende Autoimmundermatose. J Dtsch Dermatol Ges 2004; 2: 7-14

9 Dainichi T, Kurono S, Oyama B, Ishii N, Hayashi M, Shimono $C$ et al. Laminin gamma-1 is a major autoantigen in anti-p200 pemphigoid. JID 2008; 128: S20 (Abstract)

10 Rose E, Kasperkiewicz M, Rose C, Zillikens D. Bullöse Autoimmundermatosen: Neues zur Diagnostik und Therapie. Dtsch Dermatol 2007; 9: 617-623

11 Georgi M, Jainta S, Bröcker EB, Zillikens D. Autoantigene subepidermal Blasen bildender Autoimmundermatosen. Hautarzt 2001; 52: 1079-1089

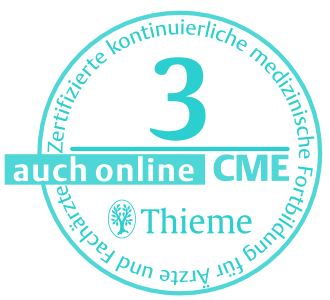




\section{CME-Fragen Diagnostik bullöser Autoimmundermatosen}

1 Welche der Aussagen zum Schleimhautpemphigoid trifft zu?

A Beim Schleimhautpemphigoid finden sich serologisch stets Autoantikörper.

B Es ist in der direkten Immunfluoreszenz durch granuläre Ablagerungen von IgG und $\mathrm{C} 3$ an der Basalmembran gekennzeichnet.

C Bei alleinigem Befall der Konjunktiven kann zur Diagnostik eine Biopsie der Mundschleimhaut für die direkte Immunfluoreszenz hilfreich sein.

D Die Autoantikörper binden in der indirekten Immunfluoreszenz mit humaner Spalthaut ausschließlich an die dermale Seite.

E Es ist obligat mit Malignomen assoziiert.

2 Welche Aussage zu immunpathologischen Befunden bei bullösen Autoimmundermatosen trifft zu?

A Bei Verdacht auf eine bullöse Autoimmundermatose sollte die direkte Immunfluoreszenz anhand einer Biopsie aus der Blase erfolgen.

B Beim bullösen Pemphigoid korreliert die Höhe der ELISAWerte von Antikörpern gegen BP230 mit der Krankheitsaktivität.

c Bei Verdacht auf eine bullöse Autoimmundermatose sollte die Gewebeprobe für die indirekte Immunfluoreszenz in einem speziellen Transportmedium versandt werden.

D Das bullöse Pemphigoid zeigt in der direkten Immunfluoreszenz eine lineare Fluoreszenz basaler Keratinozytenkerne.

E Für die histologische Untersuchung der linearen IgA-Dermatose sollte die Biopsie läsional erfolgen.

3 Welche Aussage zur Dermatitis herpetiformis Duhring trifft nicht zu?

Die Erkrankung ist obligat mit einer Zöliakie assoziiert.

B Diagnostisch wegweisend sind IgA-Autoantikörper gegen glatte Muskulatur.

C Charakteristisch ist der immunfluoreszenzoptische Nachweis granulärer Ablagerungen von IgA in der papillären Dermis der Patienten.

D Die Erkrankung zeigt histologisch ein neutrophilenreiches Infiltrat an der Junktionszone.

E Starker Juckreiz ist ein häufiges Symptom.
4 Welche Aussage zur histologischen Untersuchung von bullösen Autoimmundermatosen trifft zu?

A Bei fehlender Blasenbildung der Haut ist eine Biopsie für eine histopathologische Untersuchung nie hilfreich.

B Bei junktionaler Spaltbildung sollte differenzialdiagnostisch unter anderem an einen M. Hailey-Hailey gedacht werden.

C Eine histopathologische Untersuchung des Harnblasenepithels empfiehlt sich zum Ausschluss eines paraneoplastischen Pemphigus.

D Beim bullösen Pemphigoid ist die subepidermale Spaltbildung beim älteren Menschen in der Regel mit einer dichten Infiltration von Mastzellen assoziiert.

E Beim Pemphigus eignet sich die histopathologische Untersuchung im Gegensatz zu ELISA-Untersuchungen zum Nachweis von Antikörpern gegen Desmoglein 1 und 3 nicht gut als Verlaufsparameter.

Welche Aussage(n) zum Pemphigoid gestationis ist (sind) richtig?

1 Die Erkrankung kann auch postpartal auftreten.

2 Wie beim bullösen Pemphigoid sind die Autoantikörper gegen BP180 NC16A gerichtet.

3 Die reguläre indirekte Immunfluoreszenz auf Affenösophagus ist sensitiver als der Komplementbindungstest auf humaner Haut.

4 Die Höhe der Serumspiegel von Autoantikörpern gegen BP180 NC16A ist nicht als Verlaufsparameter geeignet.

5 Die Erkrankung verläuft meist ohne Blasenbildung.

A Keine Aussage ist richtig.

B Nur Aussage 1 ist richtig.

C Nur die Aussagen 1 und 5 sind richtig.

D Nur die Aussagen 1, 2 und 5 sind richtig.

E Nur die Aussagen 1 und 4 sind richtig.

Welche Aussage zum Pemphigus ist falsch?

A Eine Akantholyse zeigt sich beim Pemphigus foliaceus vor allem subkorneal.

B Ein positives interzelluläres Muster in der direkten Immunfluoreszenz aus befallener Mundschleimhaut spricht für die Diagnose eines Pemphigus foliaceus.

C Der Pemphigus vulgaris zeigt in der direkten Immunfluoreszenz eine interzelluläre Fluoreszenz .

D Der Pemphigus foliaceus zeigt in der indirekten Immunfluoreszenz auf Affenösophagus eine interzelluläre Fluoreszenz.

E Autoantikörper gegen Desmoglein 1 korrelieren im ELISA mit der Krankheitsaktivität beim Pemphigus foliaceus. 
A Sie ist die häufigste bullöse Autoimmundermatose im Erwachsenenalter .

B Als Autoantigen wurde ein proteolytisches Fragment von BP230 identifiziert.

C Bei Kindern finden sich in der indirekten Immunfluoreszenz IgA-Ablagerungen am Blasendach humaner Spalthaut häufiger als bei Erwachsenen.

D Immunoblot-Untersuchungen eignen sich nur in Ausnahmefällen zur Diagnosesicherung.

E Kontinuierliche granuläre IgA-Ablagerungen in den Papillenspitzen sind ein charakteristischer Befund der direkten Immunfluoreszenz.

8 Welche Aussage zum paraneoplastischen Pemphigus trifft zu?

A Die Autoantikörper sind gegen Laminin 332 gerichtet.

B Die Autoantigene werden im Epithel der Harnblase exprimiert.

C Die Erkrankung ist fakultativ mit einer Neoplasie assoziiert.

D Schleimhautläsionen sind meist gering ausgeprägt.

E In der direkten Immunfluoreszenz lassen sich IgG und C3 überwiegend in der papillären Dermis nachweisen.
9 Welche Aussage zur Epidermolysis bullosa acquisita (EBA) ist falsch?

A Die seltene IgA-EBA zeigt einen ähnlichen immunfluoreszenzoptischen Befund in der direkten Immunfluoreszenz wie die lineare IgA-Dermatose.

B Die EBA kann unter Milienbildung abheilen.

C Die EBA zeigt in der indirekten Immunfluoreszenz auf humaner Spalthaut eine Bindung von IgG-Antikörpern im Blasenboden.

D Das Zielantigen der EBA vermittelt den direkten Zell-ZellKontakt innerhalb des basalen Epithellagers.

E Die EBA kann im Immunoblot vom Anti-p200-Pemphigoid unterschieden werden.

10 Der/die Spiegel welcher im ELISA bestimmten Autoantikörperspezifitäten korreliert/korrelieren meist mit der Krankheitsaktivität?

1 Antikörper gegen Desmoglein 1

2 Antikörper gegen Kollagen Typ VII

3 Antikörper gegen Desmoglein 3

4 Antikörper gegen BP180 NC16A

5 Antikörper gegen BP230

Alle Aussagen sind richtig.

Nur die Aussagen 4 und 5 sind richtig.

Nur die Aussagen 3 und 4 sind richtig.

Nur die Aussagen 2, 3 und 4 sind richtig.

Nur die Aussagen 1, 3 und 4 sind richtig. 


\section{A Angaben zur Person}

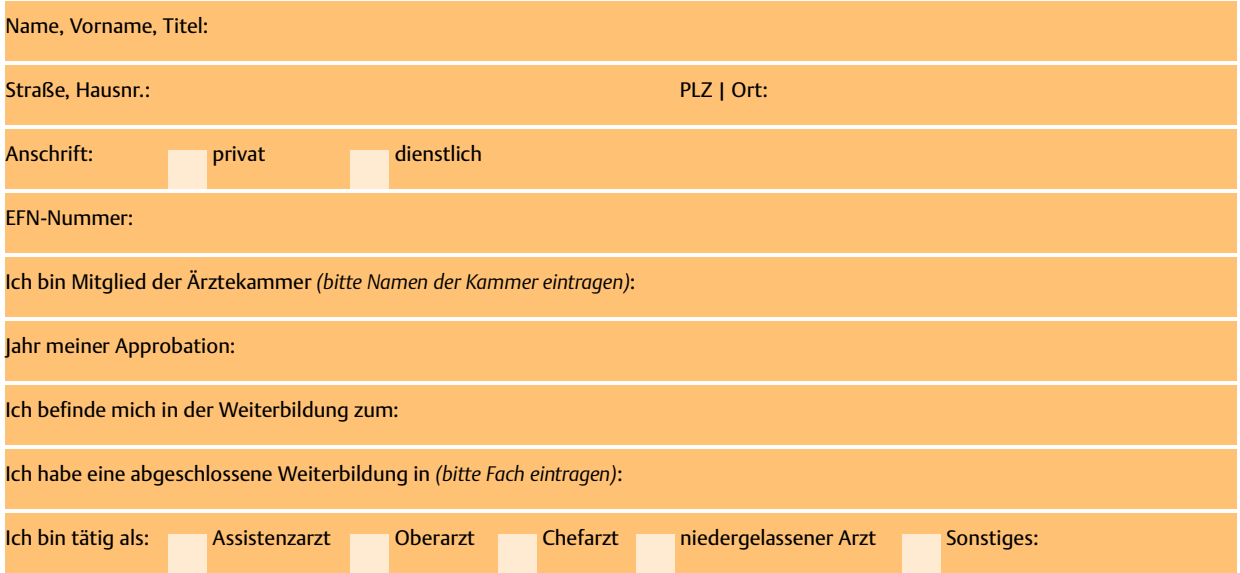

\section{B Lernerfolgskontrolle}

Bitte nur eine Antwort pro Frage ankreuzen

\section{1}

2

\section{3}

4

5

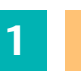

$2 A$

3 A $B$ B C D E

4 A

5 A $\quad$ B $\quad$ C D D E \begin{tabular}{l|l|l|l|l|l}
6 & A & B & $C$ & $D$ & E
\end{tabular}

\begin{tabular}{ll|l|l|l|l}
7 & A & B & C & D & E \\
\hline
\end{tabular}

8 A $B$ B $C$

9 A B C D E

10 A $B$ C

\section{Erklärung}

Ich versichere, dass ich die Beantwortung der Fragen selbst und ohne Hilfe durchgeführt habe

Ort I Datum:

Deld für CME-Wertmarke

Bitte in dieses Feld die CME-Wertmarke kleben oder Ihre Abonnement-Nummer eintragen: (siehe Adressaufkleber)

Zertifizierungsfeld (wird durch den Verlag ausgefüllt)
Unterschrift:

E Zertifizierungsfeld

\section{Ihr Ergebnis}

Sie haben von 10 Fragen richtig beantwortet.

Sie haben

bestanden und 3 CME-Punkte erworben.

nicht bestanden

Stuttgart, den

Datum

Stempel/Unterschrift

$>$ Bitte unbedingt Rückseite ausfüllen! 


\section{Fragen zur Zertifizierung}

Eine Antwort pro Frage. Bitte unbedingt ausfüllen bzw. ankreuzen, da die Evaluation sonst unvollständig ist!

\section{Didaktisch-methodische Evaluation}

1 Das Fortbildungsthema kommt in meiner ärztlichen Tätigkeit

häufig vor

regelmäßig vor selten vor gar nicht vor

2 Zum Fortbildungsthema habe ich

eine feste Gesamtstrategie

noch offene Einzelprobleme:

keine Strategie

3 Hinsichtlich des Fortbildungsthemas

fühle ich mich nach dem Studium des Beitrags in meiner Strategie bestätigt

habe ich meine Strategie verändert:

habe ich erstmals eine einheitliche Strategie erarbeitet

habe ich keine einheitliche Strategie erarbeiten können

4 Wurden aus der Sicht Ihrer täglichen Praxis heraus wichtige Aspekte des Themas nicht erwähnt?

zu knapp behandelt?

überbewertet?

\begin{tabular}{|l|l|}
\hline ja, welche \\
\hline ja, welche \\
\hline ja, welche
\end{tabular}

5 Verständlichkeit des Beitrags

Der Beitrag ist nur für Spezialisten verständlich

Der Beitrag ist auch für Nicht-Spezialisten verständlich

\section{Beantwortung der Fragen}

Die Fragen lassen sich aus dem Studium des Beitrages allein beantworten

Die Fragen lassen sich nur unter Zuhilfenahme zusätzlicher Literatur beantworten

7 Die Aussagen des Beitrages benötigen eine ausführlichere Darstellung

zusätzlicher Daten

von Befunden bildgebender Verfahren

die Darstellung ist ausreichend

8 Wie viel Zeit haben Sie für das Lesen des Beitrages und die Bearbeitung des Quiz benötigt? 